\title{
Crystal structure of hexaphenanthrolinium (hexanitrato)neodymate(III) trinitrate, $\left(\mathrm{C}_{12} \mathrm{H}_{9} \mathrm{~N}_{2}\right)_{6}\left[\mathrm{Nd}\left(\mathrm{NO}_{3}\right)_{6}\right]\left(\mathrm{NO}_{3}\right)_{3}$
}

\author{
D.-Y. Wei, H.-Z. Xie and Y.-Q. Zheng* \\ Ningbo University, Institute of Inorganic Chemistry, Municipal Key Laboratory of Solid State Chemistry, Ningbo, Zhejiang, 315211 P. R. China
}

Received April 30, 2004, accepted and available on-line August 5, 2004; CCDC no. 1267/1321
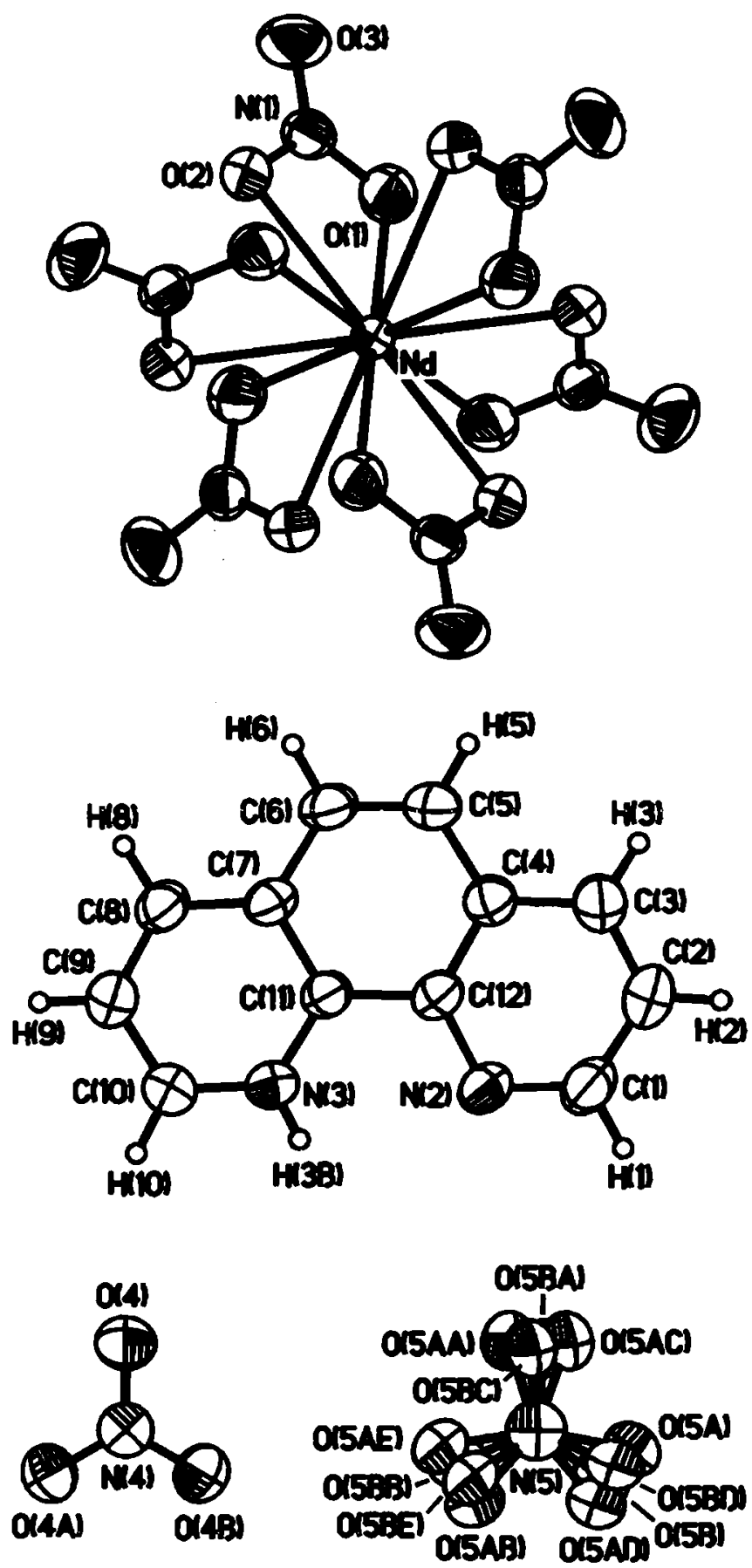

\author{
Abstract \\ $\mathrm{C}_{72} \mathrm{H}_{54} \mathrm{~N}_{21} \mathrm{NdO}_{27}$, trigonal, $R \overline{3} c$ (no. 167) \\ $a=17.5893(6) \AA, c=40.196(2) \AA, V=10770.0 \AA^{3}, Z=6$, \\ $R_{\mathrm{gt}}(F)=0.036, w R_{\text {ref }}\left(F^{2}\right)=0.101, T=293 \mathrm{~K}$.
}

\section{Source of material}

An aqueous solution of $0.15 \mathrm{~g}(1.00 \mathrm{mmol})$ adipic acid dissolved in $2 \mathrm{ml}(1 \mathrm{M}) \mathrm{NaOH}$ was added to a pale violet methanolic aqueous solution of $0.45 \mathrm{~g} \mathrm{Nd}\left(\mathrm{NO}_{3}\right)_{3} \cdot n \mathrm{H}_{2} \mathrm{O}$ and $0.20 \mathrm{~g}$ phenanthroline in $20 \mathrm{ml} \mathrm{CH} 3 \mathrm{OH} / \mathrm{H}_{2} \mathrm{O}(v / v 1 / 1)$ to give a suspension $(p H=3.93)$. Under stirring, the resulting suspension was heated for 15 minutes and then filtered out. The reddish filtrate was allowed to stand at temperature and slow evaporation afforded a small amount of reddish crystals.

\section{Experimental details}

The hydrogen atoms bonded to the carbon atoms are fixed by geometrical constraints and refined using riding modes with an overall $U_{\text {iso }}$ value, whereas $\mathrm{H} 3 \mathrm{~B}$ bonded to $\mathrm{N} 3$ atom is refined freely. The nitrate anion involving N5 atom is disordered and the population parameters for O5A and O5B sites were refined to approximately 0.25 and fixed at this value in the final refinement.

\section{Discussion}

The crystal structure of the title compound consists of nitrate anions, $\left[\mathrm{Nd}\left(\mathrm{NO}_{3}\right)_{6}\right]^{3-}$ complex anions and monoprotonated phenanthrolinium [phenH] $]^{+}$cations, in which the proton is associated to one nitrogen atom with $d(\mathrm{~N}-\mathrm{H})=0.79(3) \AA$. Within the structure two crystallographically distinct nitrate anions exist with N4 and N5 atoms at the $12 c$ and $6 a$ sites, respectively. The former nitrate group indicates a disordered tendency from the large anisotropy of the 04 atom $\left(U_{33} \approx 3 \times U_{11} \approx 3 \times U_{22}\right)$ and the latter is disordered with the nitrate oxygen atoms split over two positions $05 \mathrm{a}$ and $\mathrm{O5b}$. The phenanthrolinium cations and the complex anions are arranged in the layer parallel to (001), stabilized by weak $\mathrm{C}-\mathrm{H}$... $\mathrm{O}$ hydrogen bonding interactions. The nitrate anions are sandwiched between the layers. The NH groups of the phenanthrolinium cations form bifurcated hydrogen bonds to nitrate 04 and O5a or O5b atoms $(d(\mathrm{~N} \cdots \mathrm{O})=2.911 \AA$ and $2.946 \AA$ or $3.178 \AA$; $\angle \mathrm{O}-\mathrm{H} \cdots \mathrm{O}=147^{\circ}$ and $136^{\circ}$ or $\left.142^{\circ}\right)$. The $\left[\mathrm{Nd}\left(\mathrm{NO}_{3}\right)_{6}\right]^{3-}$ complex anions are crystallographically imposed by a $\overline{3}$ symmetry. Six chelating nitrato groups surround the central $\mathrm{Nd}$ atom to form a rare $\mathrm{NdO}_{12}$ coordination sphere [1-3] with the oxygen atoms situated at the corners of an icosahedron $(d(\mathrm{Nd}-\mathrm{O})=2.605(1) \AA$ (6x), 2.638(2) $\AA(6 x)$ ). The title Nd(III) compound is isostructural with $\left[\mathrm{Eu}\left(\mathrm{NO}_{3}\right)_{6}\right]\left[(\mathrm{Hphen})_{6}\left(\mathrm{NO}_{3}\right)_{2}\right] \mathrm{NO}_{3}[4]$, for which a disorder of the nitrate anions was not reported.

* Correspondence author (e-mail: zhengcm@nbu.edu.cn) 
Table 1. Data collection and handling.

\begin{tabular}{ll}
\hline Crystal: & reddish block, \\
& size $0.35 \times 0.47 \times 0.58 \mathrm{~mm}$ \\
Wavelength: & Mo $K_{\alpha}$ radiation $(0.71073 \AA)$ \\
$\mu:$ & $8.27 \mathrm{~cm}^{-1}$ \\
Diffractometer, scan mode: & Bruker P4, $\theta-2 \theta$ \\
$2 \theta_{\max }:$ & $70.08^{\circ}$ \\
$N(h k l)_{\text {measured, }} N(h k l)_{\text {unique: }}$ & 54597,5182 \\
Criterion for $I_{\text {obs, }} N(h k l)_{\text {gl }}:$ & $I_{\text {obs }}>2 \sigma\left(I_{\text {obs }}\right), 3950$ \\
$N\left(\right.$ param trfined: $_{\text {Programs: }}$ & 197 \\
& SHELXS-97 [5], SHELXL-97 [6] \\
\hline
\end{tabular}

Table 2. Atomic coordinates and displacement parameters (in $\AA^{2}$ ).

\begin{tabular}{llllll}
\hline Atom & Site & $x$ & $y$ & $z$ & $U_{\text {iso }}$ \\
\hline $\mathrm{H}(1)$ & $36 f$ & 0.6660 & 0.0397 & 0.0448 & $0.081(3)$ \\
$\mathrm{H}(2)$ & $36 f$ & 0.5942 & -0.1094 & 0.0520 & 0.081 \\
$\mathrm{H}(3)$ & $36 f$ & 0.4428 & -0.1877 & 0.0532 & 0.081 \\
$\mathrm{H}(5)$ & $36 f$ & 0.2993 & -0.1852 & 0.0495 & 0.081 \\
$\mathrm{H}(6)$ & $36 f$ & 0.2288 & -0.1086 & 0.0434 & 0.081 \\
$\mathrm{H}(8)$ & $36 f$ & 0.2358 & 0.0380 & 0.0358 & 0.081 \\
$\mathrm{H}(9)$ & $36 f$ & 0.3187 & 0.1883 & 0.0306 & 0.081 \\
$\mathbf{H}(10)$ & $36 f$ & 0.4707 & 0.2561 & 0.0329 & 0.081 \\
$\mathrm{H}(3 \mathrm{~B})$ & $36 f$ & $0.527(2)$ & $0.175(2)$ & $0.0368(7)$ & $0.086(8)$
\end{tabular}

Table 3. Atomic coordinates and displacement parameters (in $\AA^{2}$ ).

\begin{tabular}{|c|c|c|c|c|c|c|c|c|c|c|c|}
\hline Atom & Site & Occ. & $x$ & $y$ & $z$ & $U_{11}$ & $U_{22}$ & $U_{33}$ & $U_{12}$ & $U_{13}$ & $U_{23}$ \\
\hline Nd & $6 b$ & & 0 & 0 & 0 & $0.02967(7)$ & $U_{11}$ & $0.0475(1)$ & $0.01484(3)$ & $\mathbf{0}$ & 0 \\
\hline$O(1)$ & $36 f$ & & $-0.1148(1)$ & $-0.06870(9)$ & $0.04889(4)$ & $0.0567(8)$ & $0.0524(7)$ & $0.0617(7)$ & $0.0274(6)$ & $0.0087(6)$ & $0.0079(5)$ \\
\hline$O(2)$ & $36 f$ & & $-0.13083(8)$ & $0.02123(8)$ & $0.01746(3)$ & $0.0452(6)$ & $0.0434(6)$ & $0.0688(7)$ & $0.0238(5)$ & $0.0047(5)$ & $0.0012(5)$ \\
\hline$O(3)$ & $36 f$ & & $-0.23617(9)$ & $-0.0647(1)$ & $0.05069(4)$ & $0.0466(7)$ & $0.076(1)$ & $0.099(1)$ & $0.0216(7)$ & $0.0257(7)$ & $-0.0094(8)$ \\
\hline $\mathbf{N}(1)$ & $36 f$ & & $-0.16250(9)$ & $-0.03790(9)$ & $0.03938(4)$ & $0.0400(6)$ & $0.0408(6)$ & $0.0618(7)$ & $0.0158(5)$ & $0.0076(5)$ & $-0.0089(5)$ \\
\hline $\mathbf{N}(2)$ & $36 f$ & & $0.5647(1)$ & $0.0563(1)$ & $0.04252(4)$ & $0.0398(7)$ & $0.0619(9)$ & $0.075(1)$ & $0.0235(7)$ & $0.0010(6)$ & $0.0104(8)$ \\
\hline$N(3)$ & $36 f$ & & $0.4752(1)$ & $0.1471(1)$ & $0.03732(5)$ & $0.0403(7)$ & $0.0492(8)$ & $0.081(1)$ & $0.0178(6)$ & $0.0033(7)$ & $0.0103(7)$ \\
\hline$C(1)$ & $36 f$ & & $0.6050(1)$ & $0.0096(2)$ & $0.04554(6)$ & $0.0452(9)$ & $0.078(1)$ & $0.081(1)$ & $0.0325(9)$ & $-0.0003(8)$ & $0.010(1)$ \\
\hline $\mathrm{C}(2)$ & $36 f$ & & $0.5622(1)$ & $-0.0807(2)$ & $0.04970(5)$ & $0.063(1)$ & $0.080(1)$ & $0.069(1)$ & $0.046(1)$ & $-0.0050(9)$ & $0.002(1)$ \\
\hline$C(3)$ & $36 f$ & & $0.4726(1)$ & $-0.1271(2)$ & $0.05040(5)$ & $0.062(1)$ & $0.059(1)$ & $0.069(1)$ & $0.0346(9)$ & $-0.0055(9)$ & $-0.0006(8)$ \\
\hline$C(4)$ & $36 f$ & & $0.4261(1)$ & $-0.0814(1)$ & $0.04683(4)$ & $0.0476(8)$ & $0.0500(9)$ & $0.0524(8)$ & $0.0237(7)$ & $-0.0050(6)$ & $-0.0034(6)$ \\
\hline $\mathrm{C}(5)$ & $36 f$ & & $0.3318(1)$ & $-0.1246(1)$ & $0.04698(5)$ & $0.0467(9)$ & $0.0450(9)$ & $0.079(1)$ & $0.0153(7)$ & $-0.0043(8)$ & $-0.0063(8)$ \\
\hline$C(6)$ & $36 f$ & & $0.2898(1)$ & $-0.0790(1)$ & $0.04350(5)$ & $0.0389(7)$ & $0.0505(9)$ & $0.077(1)$ & $0.0144(7)$ & $-0.0065(7)$ & $-0.0119(8)$ \\
\hline$C(7)$ & $36 f$ & & $0.3377(1)$ & $0.0149(1)$ & $0.03985(4)$ & $0.0378(7)$ & $0.0500(8)$ & $0.0499(7)$ & $0.0184(6)$ & $-0.0050(6)$ & $-0.0060(6)$ \\
\hline $\mathrm{C}(8)$ & $36 f$ & & $0.2968(1)$ & $0.0655(1)$ & $0.03623(5)$ & $0.0426(8)$ & $0.058(1)$ & $0.067(1)$ & $0.0248(7)$ & $-0.0097(7)$ & $-0.0109(8)$ \\
\hline$C(9)$ & $36 f$ & & $0.3459(2)$ & $0.1550(2)$ & $0.03328(6)$ & $0.055(1)$ & $0.060(1)$ & $0.080(1)$ & $0.033(1)$ & $-0.0083(9)$ & $-0.002(1)$ \\
\hline$C(10)$ & $36 f$ & & $0.4367(1)$ & $0.1953(2)$ & $0.03429(6)$ & $0.054(1)$ & $0.0488(9)$ & $0.093(2)$ & $0.0230(9)$ & $0.002(1)$ & $0.010(1)$ \\
\hline$C(11)$ & $36 f$ & & $0.4297(1)$ & $0.0582(1)$ & $0.03999(4)$ & $0.0384(6)$ & $0.0469(8)$ & $0.0469(7)$ & $0.0189(6)$ & $-0.0012(5)$ & $0.0013(6)$ \\
\hline$C(12)$ & $36 f$ & & $0.4757(1)$ & $0.0102(1)$ & $0.04311(4)$ & $0.0385(7)$ & $0.0531(9)$ & $0.0462(7)$ & $0.0212(7)$ & $-0.0023(6)$ & $0.0011(6)$ \\
\hline $\mathbf{N}(4)$ & $12 c$ & & $2 / 3$ & $1 / 3$ & $0.0037(1)$ & $0.068(1)$ & $U_{11}$ & $0.067(2)$ & $0.0338(7)$ & 0 & 0 \\
\hline$O(4)$ & $36 f$ & & $0.6460(2)$ & $0.2547(2)$ & $0.00635(9)$ & $0.080(1)$ & $0.066(1)$ & $0.215(3)$ & $0.036(1)$ & $-0.011(2)$ & $-0.046(2)$ \\
\hline$N(5)$ & $6 a$ & & $2 / 3$ & $1 / 3$ & $1 / 12$ & $0.072(2)$ & $U_{11}$ & $0.059(2)$ & $0.036(1)$ & 0 & 0 \\
\hline$O(5 A)$ & $36 f$ & 0.25 & $0.5843(8)$ & $0.305(3)$ & $0.0915(8)$ & $0.060(4)$ & $0.05(1)$ & $0.148(6)$ & $0.021(6)$ & $-0.001(5)$ & $0.011(6)$ \\
\hline$O(5 B)$ & $36 f$ & 0.25 & $0.608(2)$ & $0.341(2)$ & $0.0728(8)$ & $0.060(4)$ & 0.05 & 0.148 & 0.021 & -0.001 & 0.011 \\
\hline
\end{tabular}

Acknowledgments. The project was supported by the National Natural Science Foundation of China (grant no. 20341006), the Expert Project of Key Basic Research of Ministry of Science and Technology of China (grant no. 2003CCA00800), the Zhejiang Provincial Natural Science Foundation (grant no. Z203067), the Ningbo Science and Technology Bureau (grant nos. 2003A61014, 2003A62026), the Zhejiang Provincial Fund for Analyses and Measurements (grant no. 03049) and the Scientific Research Fund of Ningbo University (grant no. Z0308007).

\section{References}

1. Deacon, G. M.; Forsyth, C. M.; Gitlits, A.; Skelton, B. W.; White, A. H.: Reactions of lanthanoid metals with 3,5-diphenylpyrazole at elevated temperature: synthesis and structure of both homoleptic, $\left[\mathrm{Ln}_{3}\left(\mathrm{Ph}_{2} \mathrm{pz}\right)_{9}\right]$ $(L n=L a, N d),\left[L n_{2}\left(P h_{2} p z\right)_{6}\right](L n=E r, L u)$, and heteroleptic, [ $\left.L n\left(\mathrm{Ph}_{2} \mathrm{pz}\right)_{3}\left(\mathrm{Ph}_{2} \mathrm{pzH}\right)_{2}\right](\mathrm{Ln}=\mathrm{La}, \mathrm{Nd}, \mathrm{Gd}, \mathrm{Tb}$, Er or $\mathrm{Yb})$, pyrazolate complexes. J. Chem. Soc., Dalton Trans. (2004) 1239-1247.

2. Lai, W.P.-W.; Wong W.-T.: Trinitrato[ $\left[N, N^{\prime}, N^{\prime \prime}\right.$-tris(2,3-dimethoxybenzamido)triethylamine]-neodymium(III). Synthesis, crystal structure and luminescence of a Nd complex containing tripodal amide ligands. New J. Chem. (2000) 943-944.
3. Ghosh, S. K.; Bharadwaj, P. K.: Self-Assembly of Lanthanide Helicate Coordination Polymer into 3D Metal-Organic Framework Stucture. Inorg. Chem. 43 (2004) 2293-2298.

4. Zhang, L.; Zhou, Y.; Xu, L.; Razak, I. A.; Chantrapromma, S.; Fun, H. K.; You, X.: Synthesis, molecular structure and fluorescence of an organicinorganic hybrid complex $\left[\mathrm{Eu}\left(\mathrm{NO}_{3}\right)_{6}\right]\left[(\mathrm{Hphen})_{6} \cdot\left(\mathrm{NO}_{3}\right)_{2}\right] \cdot \mathrm{NO}_{3}$ (phen = 1,10-phenanthroline). Inorg. Chem. Commun. 4 (2001) 368-371.

5. Sheldrick, G. M.: Phase Annealing in SHELX-90: Direct Methods for Larger Structures. Acta Crystallogr. A46 (1990) 467-473.

6. Sheldrick, G. M.: SHELXI-97. Program for the Refinement of Crystal Structures. University of Göttingen, Germany 1997. 